\title{
A WKB formalism for multicomponent fields and its application to gravitational and sound waves in perfect fluids
}

\author{
J Ehlers and A R Prasanna† \\ Max-Planck Institut für Gravitationsphysik, D-14473, Potsdam, Germany \\ Received 3 January 1996, in final form 11 April 1996
}

\begin{abstract}
We review the WKB method for multicomponent fields obeying hyperbolic linear partial differential equations and derive a general necessary and sufficient condition for the formalism to provide transport equations. We apply the method to linearized perturbations of perfect fluid solutions to Einstein's equation and show that the gravitational and sound wave modes satisfy this condition, whereas a zero-frequency, non-propagating matter mode does not. We derive the transport equations for the wave amplitudes in leading order; they exhibit in particular the influence of background curvature on the propagation of gravitational waves.
\end{abstract}

PACS numbers: $0425 \mathrm{~N}, 0430 \mathrm{~N}$

\section{Introduction}

In an earlier paper (Ehlers et al 1987) we have dealt with the problem of propagation of gravitational waves through pressureless matter using a WKB method which provided transport equations for the wave amplitudes in the zeroth order of approximation. The purpose of this paper is twofold. First we shall establish a general condition which is necessary and sufficient in order that the WKB method leads to transport equations for the amplitudes of multicomponent fields, and secondly we shall apply the method to linear perturbations of perfect fluid solutions to Einstein's field equations.

In section 2, we derive the condition announced in the preceding paragraph, which was found earlier in a special case (Ehlers et al 1987), and is based on a generalization of the long-known 'lemma on bicharacteristic directions' (Courant-Hilbert 1962, ch 6, \# 3, no 1). The method applies to linear partial differential equations of any order, provided the differential operator does not depend on the short-wave parameter to which the WKB asymptotics refers. In the context of this analysis, which is carried out in section 2, we introduce a distinction between regular and singular modes, and we separate the amplitudes of the perturbations into primary and secondary parts. In the regular case, the primary amplitudes obey transport equations along the rays while the secondary ones follow algebraically from the primary ones. Thus, initial data have to be specified for the primary amplitudes only.

In section 3, we apply the method developed in section 2 to linear perturbations of perfect fluid solutions to Einstein's field equation. The background solution is assumed to

$\dagger$ Permanent address: Physical Research Laboratory, Navrangpura, Ahmedabad 380 009, India. E-mail address: prasanna@prl.ernet.in 
have geodesic fluid world lines but is otherwise arbitrary. Naturally there are three modes. Two of them, corresponding to gravitational and sound waves, respectively, turn out to be regular, while the third one does not propagate and is singular.

The gravitational waves, in particular, propagate, according to the lowest WKB order, as in empty space; only higher orders show the influence of background curvature and, of course, 'diffraction' corrections depending on inhomogeneities. This paper may be considered as an extension of an earlier one (Ehlers et al 1987) which gave some of our results for the case of pressureless matter.

By refining the matter model and extending the approximations it should be possible to get more detailed results on the interaction of gravitational waves with matter, including damping mechanisms.

\section{General WKB formalism for vector valued functions}

Let a linear partial differential equation

$$
P(x, \partial) U=\left(A^{a b}(x) \partial_{a} \partial_{b}+B^{a}(x) \partial_{a}+C(x)\right) U=0
$$

of second order for a function $U: R^{n} \rightarrow R^{m}$ be given where the $A^{a b}, B^{a}, C$ are $(m \times m)$ matrix valued, smooth functions with real entries. (We could also consider one equation of arbitrary order $p$, but since the application in the following section concerns an equation of second order, and the general formalism is independent of the order, we treat (2.1) as a representative of the general case.)

All real solutions of (2.1) can be obtained as real parts of complex solutions; for simplicity, we denote such solutions as $U$.

In order to set up a scheme which may provide approximate solutions of (2.1) with a rapidly oscillating phase $S$ and a slowly varying amplitude $V$ one inserts, as a preliminary step, an expression

$$
U=\left[\exp \left(\frac{\mathrm{i}}{\varepsilon} S(x)\right)\right] V
$$

into (2.1). Treating $S$ and $V$ as unspecified functions and using the abbreviation

$$
l_{a}=\partial_{a} S
$$

for the wave covector, one can rearrange the expression $P\left(\mathrm{e}^{\mathrm{i} S / \varepsilon} V\right)$ as follows:

$$
P(x, \partial)\left(\mathrm{e}^{\mathrm{i} S / \varepsilon} V\right) \equiv\left(\frac{\mathrm{i}}{\varepsilon}\right)^{2} \mathrm{e}^{\mathrm{i} S / \varepsilon}\left(L_{0}+\frac{\varepsilon}{\mathrm{i}} L_{1}+\left(\frac{\varepsilon}{\mathrm{i}}\right)^{2} P\right) V
$$

where

$$
L_{0}(x, l)=A^{a b}(x) l_{a} l_{b}
$$

is called the principal symbol of $P$, and

$$
L_{1}(x, l, \partial)=2 A^{a b}(x) l_{a} \partial_{b}+A^{a b}(x) \partial_{a} l_{b}+B^{a}(x) l_{a}
$$

is a first order differential operator.

As in the typical case, considered here, so in the general case of a $p$ th order operator, the principal parts of $L_{0}, \ldots, L_{p}$ (defined as in (2.4)) are determined by the principal part of the original operator and one has formulae similar to (2.5)-(2.7). In particular, the principal part $L_{1}^{(1)}$ of $L_{1}$ is always related to $L_{0}$ by

$$
L_{1}^{(1)}=\left(\frac{\partial}{\partial l_{a}} L_{0}\right) \partial_{a} .
$$


$L_{j}$ is of order $j$ and depends on $l$ and its derivatives up to order $j$ as long as $j<p-1$.

To construct formal solutions of (2.1) in a step by step procedure, one now susbstitutes for $V$ a series

$$
V(x, \varepsilon) \sim \sum_{n=0}^{\infty}\left(\frac{\varepsilon}{\mathrm{i}}\right)^{n} V_{n}(x)
$$

and requires the terms of order $1, \varepsilon, \varepsilon^{2}, \ldots$ in the resulting equation

$$
\left(L_{0}+\frac{\varepsilon}{\mathrm{i}} L_{1}+\left(\frac{\varepsilon}{\mathrm{i}}\right)^{2} P\right)\left(V_{0}+\frac{\varepsilon}{\mathrm{i}} V_{1}+\cdots\right)=0
$$

to vanish separately. The lowest 'zeroth' order equation

$$
L_{0} V_{0}=0
$$

admits nontrivial solutions $V_{0}$ if and only if $S$ obeys the characteristic equation

$$
\operatorname{det} L_{0}=\operatorname{det}\left(A^{a b}(x) l_{a} l_{b}\right):=Q(x, l)=0 .
$$

$Q$ is a homogeneous polynomial of degree $2 m$ in the variables $l_{a}$ whose coefficients depend on $x$; it is called the characteristic form of the differential operator $P$.

Geometrically, $Q$ may be viewed as a function on phase space $\pi \equiv\{(x, l)\}$. In general, the so-called characteristic set of (real) points of $\pi$ which obey (2.11) consists of several hypersurfaces (branches) which may intersect or touch each other. Let us henceforth assume that the equation

$$
H(x, l)=0
$$

describes locally over some domain of $R^{n}$, one such hypersurface $\Sigma$ and let us suppose that

$$
\text { on } \Sigma: \frac{\partial H}{\partial l} \neq 0 \quad \operatorname{rank} L_{0}=r=\text { constant. }
$$

Then we say that $\Sigma$ corresponds to a simple mode, that (2.12) is its dispersion relation and

$$
H(x, \mathrm{~d} S)=0
$$

its eikonal equation. (Note that what matters is not the function $H$, but the hypersurface $\Sigma$ in $\pi$ defined by (2.14).)

On $\Sigma, L_{0}$ admits $p=m-r$ linearly independent left null vector fields $\lambda_{j}$ and as many right null vector fields $\rho^{j}$,

$$
\lambda_{j} L_{0}=0 \quad L_{0} \rho^{j}=0 \quad(1 \leqslant j \leqslant p) .
$$

Let $\left(\lambda_{1}, \ldots, \lambda_{p}, \tilde{\lambda}_{p+1}, \ldots, \tilde{\lambda}_{m}\right)$ and $\left(\rho^{1}, \ldots, \rho^{p}, \tilde{\rho}^{p+1}, \ldots, \tilde{\rho}^{m}\right)$ each be a basis (in the appropriate linear space). We may then write

$$
V_{n}=a_{j}^{(n)} \rho^{j}+b_{j}^{(n)} \tilde{\rho}^{j}=: V_{n}^{(1)}+V_{n}^{(2)} .
$$

We shall see that the two terms of $V_{n}$ play different parts in the WKB expansion; therefore we call $V_{n}^{(1)}$ the primary, $V_{n}^{(2)}$ the secondary amplitude of $n$th order. (This decomposition depends, of course, on the mode considered as well as on the chosen basis $\rho$.) The zeroorder equation (2.10) then requires

$$
V_{0}=V_{0}^{(1)}=a_{j}^{(0)} \rho^{j} \quad\left(\Rightarrow b_{j}^{(0)}=0\right),
$$

thus $V_{0}$ is independent of the choice of the basis $\rho$. 
In the case of waves (2.17), the kernel of $L_{0}$ is the $p$-dimensional space of polarization states. The assumptions (2.13) which we made about $\Sigma$ imply that on $\Sigma$, there exists a $p \times p$ matrix valued function $M_{j}^{k}$ such that

$$
\lambda_{j}\left(\frac{\partial L_{0}}{\partial l_{a}}\right) \rho^{k}=M_{j}^{k} \frac{\partial H}{\partial l_{a}} .
$$

This generalized lemma on bicharacteristic directions is crucial for the derivation of transport equations for the primary amplitudes within the WKB method. (For a proof, see Ehlers et al (1987).) Note that the term 'simple mode' was used differently in that paper than it is here, which, however, does not affect the argument. We call a simple mode regular if $M$ is invertible, otherwise the mode is said to be singular.

We continue the review of the WKB formalism. First, one has to solve the eikonal equation (2.14). That can be done (locally) via Hamilton's ordinary differential equations (method of ray tracing):

$$
\dot{x}^{a}=\frac{\partial H}{\partial l_{a}} \quad i_{a}=-\frac{\partial H}{\partial x^{a}} .
$$

Suppose $S$ is a real solution of (2.15). It determines a ray bundle in $x$-space generated by the vector field

$$
T^{a}:=\frac{\partial H}{\partial l_{a}}\left(x^{b}, \partial_{a} S\right)
$$

The first order WKB equation resulting from (2.10) requires

$$
L_{0} V_{1}+L_{1} V_{0}=0 \text {. }
$$

Due to the definition of $\lambda_{j},(2.15)$ and (2.17), this equation admits a solution $V_{1}$ if and only if for $1 \leqslant j \leqslant p$,

$$
\lambda_{j} L_{1} V_{0}=\lambda_{j} L_{1} V_{0}^{(1)}=\lambda_{j} L_{1}\left(\rho^{k} a_{k}^{(0)}\right)=0 .
$$

Because of (2.7), (2.18) and the definition (2.20) of the transport vector field $T^{a}$ associated with $S$, (2.22) has the form

$$
\left(M_{j}^{k} T^{a} \partial_{a}+Q_{j}^{k}\right)\left(a_{k}^{(0)}\right)=0,
$$

where

and

$$
M_{j}^{k} T^{a}:=M_{j}^{k} \frac{\partial H}{\partial l_{a}}=\lambda_{j} \frac{\partial L_{0}}{\partial l_{a}} \rho^{k}
$$

$$
Q_{j}^{k}:=\lambda_{j}\left(\frac{\partial L_{0}}{\partial l_{a}} \partial_{a} \rho^{k}-\left(A^{a b} \partial_{a} l_{b}+B^{a} l_{a}\right) \rho^{k}\right) .
$$

If the mode is regular, this equation is equivalent to a first order, linear, homogeneous ordinary differential equation for the lowest order amplitude $V_{0}$ called its transport equation. It determines $V_{0}$ everywhere, given arbitrary initial data on some hypersurface in $x$-space intersecting the rays. Moreover (2.21) then determines $V_{1}^{(2)}$ algebraically in terms of $V_{0}$ and its first derivatives.

The second order equation requires

$$
L_{0} V_{2}+L_{1} V_{1}+P V_{0}=0 .
$$

Its solvability for $V_{2}$ requires via (2.8) and (2.19) the inhomogeneous transport equation

$$
\lambda_{j} L_{1} V_{1}^{(1)}=-\lambda_{j} L_{1} V_{1}^{(2)}-\lambda_{j} P V_{0}
$$


for $V_{1}^{(1)}$, the right-hand side being known already. If that equation has been solved, (2.24) gives $V_{2}^{(2)}$ etc. Note that, generally, the transport equation for $V_{n}^{(1)}$ is necessary and sufficient in order that the $(N+1)$ th order equation admits a (unique) solution $V_{n+1}^{(2)}$. (This is a special case of a more general feature of perturbation theory: an $n$th order solution is 'reliable', i.e. extendable to higher order, only if the former obeys an equation which frequently is an evolution equation; more complicated examples are post-Newtonian or post-Minkowskian equations of motion in GR.) Note also that, given a phase function $S$, initial data have to be prescribed only for the primary amplitudes of all orders. In practice, one will usually set the initial values for all amplitudes but the lowest order one to zero, in order that the lowest order already approximates the full solution as well as possible near the initial hypersurface. After $n$ steps one obtains a formal solution of (2.1):

$$
\mathrm{e}^{\mathrm{i} S / \varepsilon}\left(V_{0}+\frac{\varepsilon}{\mathrm{i}} V_{1}+\cdots+\left(\frac{\varepsilon}{\mathrm{i}}\right)^{n-1} V_{n-1}\right)
$$

to within order $\varepsilon^{n-1}$. If the original equation is hyperbolic, the one-parameter family (2.26) of functions is asymptotic for $\varepsilon \rightarrow 0$ to a one-parameter family of solutions of (2.1), the 'error' being of order $\varepsilon^{n}$ (see, for example, Courant-Hilbert 1962, Taylor 1981). In applications it is usual to put $\varepsilon=1$ at the end of the calculations. The role of the small parameter is then played by $\lambda / L$ where $\lambda$ is the scale on which $S$ varies (typical 'wavelength') and $L$ is the scale on which the coefficient functions of (2.1), the 'background field', vary; in general, the $V_{n}$ will vary on this scale also.

In the singular case (2.23) will, in general, impose further 'algebraic' restrictions on $V_{0}^{(1)}$ obtained by multiplying (2.23) with the left null vector $\mu_{l}$ of $M$

$$
\mu_{l}^{j} Q_{j}^{k}\left(a_{k}^{(0)}\right)=0
$$

In the extreme case $M_{j}^{k}=Q_{j}^{k}=0$, (2.21) only gives $V_{1}^{(2)}$ in terms of $V_{0}$ and its first derivatives and (2.25) provides an underdetermined system of second order partial differential equations for $V_{0}^{(1)}$ which is more complicated than the original equation (2.1) for $U$, so that in this case the WKB formalism is useless; in particular, it does not lead to transport equations for the leading amplitude $V_{0}$. The example treated in the next section may suggest that this extreme singular case occurs for zero frequency, non-propagating modes only. Whether that is true is by no means obvious from the general formalism; it should be tested in other cases. We have not investigated the general singular case and are not aware of a case where it occurs.

The formalism described above can without change be applied if $U$ in (2.1) denotes a tensor field and $\partial_{a}$ is replaced by the covariant derivative $\nabla_{a}$.

\section{Short wave linear perturbations of perfect fluid solutions of Einstein's equations}

The linear perturbation equations of a background solution $\left(g_{a b}, U^{a}, \rho\right)$ of Einstein's equations with a cosmological constant $\Lambda$, for a perfect fluid satisfying an equation of state $p=p(\rho)$ are given by

$$
\begin{aligned}
& \hat{\rho}=\frac{1}{\left(1+3 C_{s}^{2}\right)}\left[2 \hat{R}_{i j}+(\rho+3 p-2 \Lambda) \hat{g}_{i j}\right] U^{i} U^{j} \\
& \hat{p}=C_{s}^{2} \hat{\rho} \\
& \rho \hat{U}_{k}=\left[\hat{R}_{i j} h_{k}^{i}+\frac{1}{2}(\rho-p+2 \Lambda) \hat{g}_{k j}\right] U^{j}
\end{aligned}
$$


and

$$
\begin{aligned}
H_{a b}^{i j}\left(2 \delta_{(i}^{c} \nabla_{j)}^{d}\right. & \left.-\delta_{i}^{c} \delta_{j}^{d} \nabla^{2}-g^{c d} \nabla_{i} \nabla_{j}\right)\left(\hat{g}_{c d}\right) \\
& \left.=\left[(\rho-p+2 \Lambda) h_{a}^{i} h_{b}^{j}-(\rho+3 p-2 \Lambda) \alpha h_{a b} U^{i} U^{j}\right] \hat{g}_{i j}\right) .
\end{aligned}
$$

Here the notation is as follows. Overhead hats denote the perturbed quantities; $C_{s}^{2}=\frac{\mathrm{d} p}{\mathrm{~d} \rho}$ is the squared sound speed; the speed of light and the Einstein gravitational constant are set equal to 1 . The signature of the metric is $+2, h_{a}^{i}=\delta_{a}^{i}+U^{i} U_{a}$ is the usual projection tensor and

$$
H_{a b}^{i j}=h_{a}^{i} h_{b}^{j}-\alpha h_{a b} U^{i} U^{j} .
$$

The scalar $\alpha:=\left(1-C_{s}^{2}\right) /\left(1+3 C_{s}^{2}\right)$ takes values in the range $0 \leqslant \alpha \leqslant 1$ since $1 \geqslant C_{s} \geqslant 0$.

As was shown in Ehlers et al (1987) one can impose the gauge condition $\hat{g}_{a b} U^{b}=0$, if the streamlines of the background fluid are geodesics. We assume that and can thus use that gauge condition as in the earlier paper and simplify the system of equations (3.3) as follows:

$$
\begin{gathered}
P_{a b}^{i j} \hat{g}_{i j} \equiv\left[\left(2 h_{(a}^{i} h_{b)}^{c} \nabla^{j} \nabla^{c}-h_{a}^{i} h_{b}^{j} \nabla^{2}-g^{i j} h_{a}^{c} h_{b}^{d} \nabla_{c} \nabla_{d}\right)\right. \\
+\alpha h_{a b}\left(g^{i j} \nabla_{u}^{2}+2 \nabla^{(i} U^{j)} \nabla_{u}+4\left(\nabla_{d} U^{i} \nabla^{[d} U^{j]}\right)\right) \\
\left.-(\rho-p+2 \Lambda) h_{a}^{i} h_{b}^{j}\right]\left(\hat{g}_{i j}\right)=0 .
\end{gathered}
$$

This equation for $\hat{g}_{i j}$ governs the linearized perturbations under the conditions specified above; $\hat{\rho}$ and $\hat{U}_{k}$ are as given in (3.1) and (3.2).

Equation (3.4) is of the form (2.1) (except for the substitution of $\nabla_{a}$ in place of $\partial_{a}$ ), whence the formalism of section 2 can be applied. Using equations (2.5) and (2.6), we obtain the operators $L_{j}$ corresponding to (3.4) which act on the six-dimensional space of metric perturbations as follows:

$$
\begin{aligned}
& L_{0 a b}^{c d}:=-2 h_{(a}^{(c} k^{d)} k_{b)}+h_{(a}^{(c} h_{b)}^{d)} l^{2}+\left(k_{a} k_{b}-\alpha \omega^{2} h_{a b}\right) h^{c d}, \\
& \begin{array}{c}
L_{1 a b}^{c d}:=\left[-4 h_{(a}^{i} h_{b)}^{(c}\left(l^{d)} \nabla_{i}+\nabla^{d)} l_{i}\right)+2 h_{(a}^{(c} k_{b)} \nabla^{d)}+2 h_{(a}^{(c} h_{b)}^{d)}\left(\nabla_{l}+\theta / 2\right)\right. \\
+2 \alpha \omega h_{a b} \nabla^{d} U^{c}-h^{c d}\left\{h_{(a}^{i} h_{b)}^{j} \nabla_{j} l_{i}+2 h_{(a}^{i} k_{b)} \nabla_{i}\right. \\
\left.\left.\quad-\alpha h_{a b}\left(2 \omega \nabla_{u}-U^{i} \nabla_{u} l_{i}\right)\right\}\right],
\end{array}
\end{aligned}
$$

where $l_{a}=\partial_{a} S$ as in (2.3) and $\omega:=-U^{a} l_{a}, k_{a}=h_{a}^{b} l_{b}, \theta=\nabla^{a} l_{a}, \nabla_{u}=U^{i} \nabla_{i}, \nabla_{l}=l^{i} \nabla_{i}$.

In analogy to (2.2), (2.8) we use the notations $v_{i j}, v_{(0) i j}$ for the total and partial complex amplitudes of $\hat{g}_{i j}$.

As has been noticed earlier (Ehlers et al 1987), the characteristic equation in this case is

$$
\left|\operatorname{det} L_{0}\right|=l^{4} \omega^{6}\left[\omega^{2}-C_{s}^{2} k^{2}\right]=0
$$

or equivalently

$$
\left(g^{a b} l_{a} l_{b}\right)^{2}\left[\left(U^{a} U^{b}-C_{s}^{2} h^{a b}\right) l_{a} l_{b}\right]\left(U^{a} l_{a}\right)^{6}=0 .
$$

Thus there are three modes:

(i) the gravitational wave mode, given by the Hamiltonian $H=\frac{1}{2} g^{a b} l_{a} l_{b}$ and the null geodesic rays with tangent $T^{a}=l^{a}$;

(ii) the sound wave mode, given by $H=\frac{1}{2}\left[C_{s}^{2} h^{a b}-U^{a} U^{b}\right] l_{a} l_{b}$ and the sound rays with tangent $T^{a}=\omega\left(\frac{C_{s} k^{a}}{k}+U^{a}\right)$; and

(iii) the matter mode given by $H=U^{a} l_{a}$ and 'matter rays' with tangent $T^{a}=U^{a}$. 


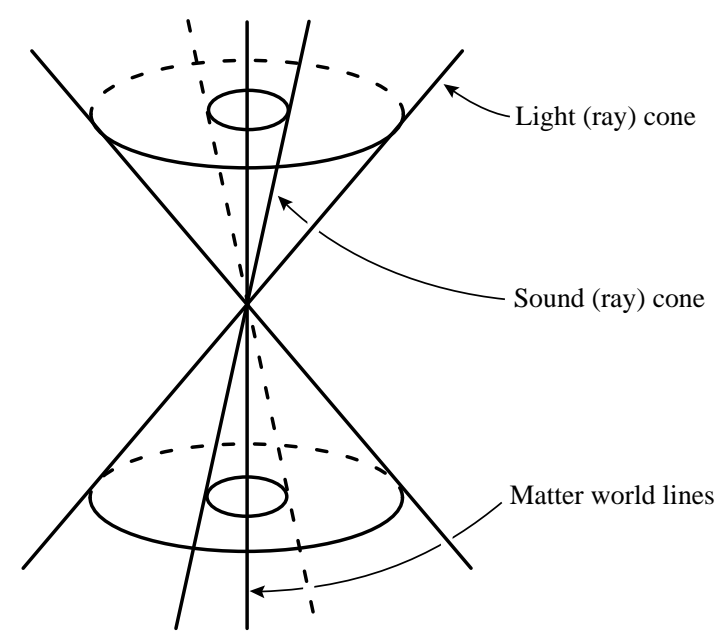

Figure 1.

Thus the (zero frequency) excitations of the last mode do not propagate relative to the unperturbed matter, in contrast to those of the other two.

Assuming $\left(e_{i}^{a}\right) i=1,2,3$ to denote an orthonormal basis in the space orthogonal to $U^{a}$, with $e_{3}^{[a} k^{b]}=0$, one can write explicitly the basis vectors in the space of amplitudes and its dual respectively according to (2.15), as follows.

Mode (i), $l^{2}=0, \operatorname{rank} L_{0}=4, p=2$

$$
\begin{aligned}
& \lambda_{1}=e_{+}^{a b}:=\left(e_{1}^{a} e_{1}^{b}-e_{2}^{a} e_{2}^{b}\right) \quad \lambda_{2}=e_{\times}^{a b}:=2 e_{1}^{(a} e_{2}^{b)}, \\
& \tilde{\lambda}_{3}=e_{1}^{a b}:=2 e_{1}^{(a} e_{3}^{b)} \quad \tilde{\lambda}_{4}=e_{2}^{a b}:=2 e_{2}^{(a} e_{3}^{b)}, \\
& \tilde{\lambda}_{5}=e_{3}^{a b}:=e_{3}^{a} e_{3}^{b} \quad \tilde{\lambda}_{4}=e_{4}^{a b}:=\left(e_{1}^{a} e_{1}^{b}+e_{2}^{a} e_{2}^{b}\right)
\end{aligned}
$$

and

$$
\begin{array}{ll}
\rho_{1}=e_{a b}^{+} & \rho_{2}=e_{a b}^{\times}, \\
\tilde{\rho}_{3}=e_{a b}^{1} & \tilde{\rho}_{4}=e_{a b}^{2}, \\
\tilde{\rho}_{5}=e_{a b}^{3} & \tilde{\rho}_{6}=e_{a b}^{4} .
\end{array}
$$

Mode (ii), $\omega^{2}=C_{s}^{2} k^{2}, \operatorname{rank} L_{0}=5, p=1$

$$
\begin{array}{ll}
\lambda_{1}=\left(\left(1+C_{s}^{2}\right) h^{a b}-2 e_{3}^{a b}\right) & \tilde{\lambda}_{2}=e_{+}^{a b}, \\
\tilde{\lambda}_{3}=e_{\times}^{a b} & \tilde{\lambda}_{4}=e_{1}^{a b}, \\
\tilde{\lambda}_{5}=e_{2}^{a b} & \tilde{\lambda}_{6}=e_{3}^{a b}
\end{array}
$$

and

$$
\begin{array}{ll}
\rho^{1}=\left(C_{s}^{2} h_{a b}+e_{a b}^{3}\right) & \tilde{\rho}_{2}=e_{a b}^{+}, \\
\tilde{\rho}_{3}=e_{a b}^{\times} & \tilde{\rho}_{4}=e_{a b}^{1}, \\
\tilde{\rho}_{5}=e_{a b}^{2} & \tilde{\rho}_{6}=e_{a b}^{3} .
\end{array}
$$

Mode (iii), $\omega^{2}=0, \operatorname{rank} L_{0}=3$

$$
\begin{aligned}
& \lambda_{1}=e_{1}^{a b} \quad \lambda_{2}=e_{2}^{a b}, \\
& \lambda_{3}=h^{a b}-2 e_{3}^{a b} \quad \tilde{\lambda}_{4}=e_{+}^{a b}, \\
& \tilde{\lambda}_{5}=e_{\times}^{a b} \quad \tilde{\lambda}_{6}=e_{3}^{a b}
\end{aligned}
$$


and

$$
\begin{array}{ll}
\rho_{1}=e_{a b}^{1} & \rho_{2}=e_{a b}^{2}, \\
\rho_{3}=e_{a b}^{3} & \tilde{\rho}_{4}=e_{a b}^{+}, \\
\tilde{\rho}_{5}=e_{a b}^{x} & \tilde{\rho}_{6}=e_{a b}^{4} .
\end{array}
$$

Accordingly the lowest order amplitudes of $\hat{g}_{i j}$ are

$$
\text { (i) } \quad v_{0 a b}^{(1)}=a_{+}^{(0)} e_{a b}^{+}+a_{\times}^{(0)} e_{a b}^{\times},
$$

which is transverse and trace free;

(ii) $\quad v_{0 a b}^{(1)}=b\left(C_{s}^{2} h_{a b}+e_{a b}^{3}\right)$,

with trace $v_{0}^{(1)} \underset{a}{a}=\left(1+3 C_{s}^{2}\right) b$;

$$
\text { (iii) } \quad v_{0 a b}^{(1)}=a_{1}^{(0)} e_{a b}^{1}+a_{2}^{(0)} e_{a b}^{2}+a_{3}^{(0)} e_{a b}^{3}=A_{(a} e_{b)}^{3},
$$

with $A_{a}=a_{1}^{(0)} e_{a}^{1}+a_{2}^{(0)} e_{a}^{2}+a_{3}^{(0)} e_{a}^{3}$ arbitrary, and

$$
v_{0}^{(1)} \underset{a}{a}=a_{3}^{(0)} \text {. }
$$

One can now compute the matrices $M_{j}^{k}$, using the definition (2.24). It turns out that for gravitational waves $M_{j}^{k}$ is the unit matrix, while for sound waves $M$ is the positive scalar $2 /(1+\alpha)=\left(1+3 C_{s}^{2}\right) /\left(1+C_{s}^{2}\right)$, and for the zero frequency matter mode $M_{j}^{k}$ vanishes identically.

For the three particular cases of special interest the situation is as follows. In the case of dust $(p=0), C_{s}=0$ or $\alpha=1$, there are no sound waves; in fact for $C_{s}=0$, mode (ii) degenerates into the longitudinal part of mode (iii). In the case of stiff matter $(\rho=p)$, $C_{s}=1$ and $\alpha=0$, sound waves propagate with the speed of light but are (of course) volume changing, in contrast to gravitational waves, and $M=2$. For the case of pure radiation, $p=\frac{1}{3} \rho, C_{s}^{2}=\alpha=\frac{1}{3}$ and $M=\frac{3}{2}$. According to the definition given in section 2, we thus have the modes corresponding to gravitational waves and to sound waves, regular, whereas the matter mode is singular.

We now consider the transport equation for the amplitudes of the regular modes.

\subsection{The gravitational wave mode}

In this case, as has been shown earlier, the transport of the primary amplitudes $a_{+}^{(0)}$ and $a_{\times}^{(0)}$ is governed by the equations

$$
\left(\nabla_{l}+\frac{\theta}{2}\right)\left(\begin{array}{c}
a_{+}^{(0)} \\
a_{\times}^{(0)}
\end{array}\right)=0,
$$

where the vectors $e_{1}^{a}$ and $e_{2}^{a}$ in (3.7) are assumed to be quasi-parallelly transported $\dagger$ along the rays, null geodesics with tangents $l^{a}$. Applying the method of section 2, we find the structure of the first order secondary amplitude

$$
v_{1}^{(2)}=\frac{2}{k}\left(e_{1}^{c} \tilde{\rho}_{3}+e_{2}^{c} \tilde{\rho}_{4}\right)\left(\nabla^{d} v_{0 c d}\right) .
$$

† Quasi-parallel transport has been defined in Ehlers et al (1987). 
This part of $v_{1}$ is thus smaller than $v_{0}$ by a factor of order $\frac{\lambda}{L}$, as expected. In contrast to the primary amplitude, its polarization is neither longitudinal nor transverse, but mixed. The transport equation for these first order primary amplitudes is then given by

$$
\begin{aligned}
\left(\nabla_{l}+\frac{\theta}{2}\right)\left(a_{+}^{(1)}\right) & =\frac{1}{2}(\rho-p+2 \Lambda)\left(a_{+}^{(0)}\right) \\
+ & \frac{1}{2} e^{i j}\left\{\left[2\left(\nabla^{d} \nabla_{i}+\nabla_{i} \nabla^{d}\right) \delta_{j}^{c}-\delta_{i}^{c} \delta_{j}^{d} \nabla^{2}-h^{c d} \nabla_{j} \nabla_{i}\right]\left(v_{0 c d}\right)\right\}
\end{aligned}
$$

and a similar one for $a_{\times}^{(1)}$.

Using the definitions of the curvature tensor $\left(R_{i j k}^{h}\right)$ and the Weyl tensor $\left(C_{i j k}^{h}\right)$ and the field equation

$$
R_{i}^{j}=\frac{1}{2} R \delta_{i}^{j}=(\rho+p) U_{i} U^{j}+p \delta_{i}^{j}-\Lambda \delta_{i}^{j}
$$

one can rewrite (3.19) as

$$
\begin{gathered}
\left(\nabla_{l}+\frac{\theta}{2}\right)\left(a_{+}^{(1)}\right)=\frac{1}{2} e_{+}^{i j}\left\{\left[4 \nabla_{i} \nabla^{h} v_{0 j h}-\nabla^{2} v_{0 i j}-h^{c d} \nabla_{j} \nabla_{i} v_{0 c d}\right]+2 C_{h i j} v_{0}^{h k}\right\} \\
-\frac{1}{2}(\rho-3 p+4 \Lambda) a_{+}^{(0)} .
\end{gathered}
$$

If the background spacetime is conformally flat, we have $C^{h} \underset{i j}{k}=0$, and the transport equations for $a_{+}^{(1)}$ and $a_{\times}^{(1)}$ turn out to be

$$
\left(\nabla_{l}+\frac{1}{2} \theta\right)\left(a_{+}^{(1)}\right)+\frac{R}{3}\left(a_{+}^{(0)}\right)=\frac{1}{2} e_{+}^{i j}\left(4 \nabla_{i} \nabla^{c} \delta_{j}^{d}-\delta_{i}^{c} \delta_{j}^{d} \nabla^{2}-h^{c d} \nabla_{j} \nabla_{i}\right)\left(v_{0 c d}\right),
$$

which exhibits the influence of both the background curvature $R$ and the inhomogeneities of $v_{0}$ on the wave.

\subsection{Sound waves}

We next consider mode (ii), $\omega^{2}=C_{s}^{2} k^{2}$, representing the sound waves, whose primary amplitude is given by (3.14),

$$
v_{0 c d}=b\left(c_{s}^{2} h_{c d}+e_{c d}^{3}\right) \text {. }
$$

Using this in equation (2.21) and left multiplying this with $\lambda$ from (3.9), one gets after simplification the transport equation for $b$,

$$
\begin{aligned}
4 C_{s}^{2}\left[\left(\nabla_{T}+\eta\right) b\right] & +\left\{\left(1+C_{s}^{2}\right)\left(1+3 C_{s}^{2}\right)\left(\nabla_{a} k^{a}-\omega \nabla_{a} U^{a}\right)\right. \\
+ & {\left[\left(1+C_{s}^{2}\right) h^{i j}-e_{3}^{i j}\right]\left[\left(1-C_{s}^{2}\right)\left(\nabla_{i} k_{j}\right)-\left(1+3 C_{s}^{2}\right)\left(\nabla_{i} \omega\right) U_{j}\right] } \\
- & {\left[\left(1+2 C_{s}^{2}+5 C_{s}^{4}\right) h^{i j}-4\left(1+C_{s}^{2}\right) e_{3}^{i j}\right] \omega\left(\nabla_{i} U_{j}\right) } \\
+ & \left.4\left(C_{s}^{2} h_{i j}+e_{i j}^{3}\right)\left[\nabla_{l}\left(C_{s}^{2} h^{i j}-e_{3}^{i j}\right)\right]\right\}(b)=0,
\end{aligned}
$$

where $\eta=\nabla_{a} T^{a}, \nabla_{T}=T^{a} \nabla_{a}, T^{a}=\left(C_{s}^{2} k^{a}+\omega U^{a}\right)$ is the tangent vector to the sound rays.

Finally using (3.1) and (3.2), along with the gauge condition $\hat{g}_{a b} U^{b}=0$, one can find the density and the 4-velocity perturbation to be

$$
\begin{gathered}
\hat{\rho}=\frac{2}{\left(1+3 C_{s}^{2}\right)}\left\{\left(\varepsilon^{-2} \omega^{2}-2 \mathrm{i} \varepsilon^{-1} \nabla_{u} \omega\right) v^{T}-2 \nabla_{u}\left(v_{j k} \nabla^{(k} U^{j)}\right)-\nabla_{u}^{2} v^{T}\right. \\
\left.-2 v_{i j} \nabla_{a} U^{i} \nabla^{a} U^{j}+2 R_{k i j l} U^{i} U^{j} v^{k l}\right\}
\end{gathered}
$$


and

$$
\begin{gathered}
\rho \hat{U}_{a}=\varepsilon^{-2}\left(\omega l^{m} v_{a m}\right)+\mathrm{i} \varepsilon^{-1}\left[U^{j} l^{m} \nabla_{a} v_{j m}+l^{m} U_{a} \nabla_{u}\left(U^{j} v_{j m}\right)+U^{j} k_{a} \nabla^{m} v_{j m}\right. \\
+l^{m} \nabla_{u} v_{a m}-\omega h_{a}^{i} \nabla^{m} v_{i m}+U^{j}\left(\nabla^{m} l_{j}\right) v_{a m}-2 U^{j} h_{a}^{i} \nabla_{l} v_{i j} \\
\left.\quad-\left(\nabla_{u} k_{a}\right) v^{T}-\omega \nabla_{a} v^{T}-l_{a} \nabla_{u} v^{T}\right] \\
+h_{a}^{i} U^{j}\left(\nabla^{m} \nabla_{i} v_{j m}+\nabla^{m} \nabla_{j} v_{i m}-\nabla^{2} v_{i j}\right)-h_{a}^{i} \nabla_{u} \nabla_{i} v^{T},
\end{gathered}
$$

where a superscript $T$ represents the trace $v_{a}^{a}$ and $\nabla^{(k} U^{j)}$ the rate of deformation of the background matter flow. It is seen that for the gravitational wave mode $\left(l^{2}=0, v_{0 a b}=e_{a b}^{+}\right.$ or $\left.e_{a b}^{\times}\right)$and the matter mode $\left(\omega=0, v_{0 a b}=A_{(a} k_{b}\right)$ both $\hat{\rho}$ and $\hat{U}_{a}$ vanish in the leading $\left(\varepsilon^{-2}\right)$ order whereas for sound waves $\left(\omega^{2}=C_{s}^{2} k^{2}\right)$

$$
\hat{\rho}=2 b \omega^{2}
$$

and

$$
\rho \hat{U}_{a}=b\left(1+C_{s}^{2}\right) \omega k_{a}
$$

in the leading order.

From the general equations (3.1) to (3.3), it may easily be seen that the treatment used above is equally valid for the vacuum case, $R_{i j}=0$, as there appear no terms involving $\rho, p$ or $\Lambda$ in the leading order equations, i.e. the dispersion relation and the transport equations for the primary amplitude, and $\hat{\rho}$ vanishes to the order required.

\section{Acknowledgments}

ARP would like to thank the authorities of the Max-Planck Institut für Gravitationsphysik for the hospitality and the excellent atmosphere provided during his visit (July 1995). We wish to thank Mr V T Viswanathan (PRL) for speedy and neat typing of the manuscript.

\section{References}

Courant R and Hilbert D 1962 Methods of Mathematical Physics vol 2 (New York: Interscience)

Ehlers J, Prasanna A R and Breuer R 1987 Class. Quantum Grav. 4253

Taylor M R 1981 Pseudo Differential Operators (Princeton, NJ: Princeton University Press) 